\title{
A Rare Case of Sacral Pott's Disease
}

\author{
Case Report
}

Volume 2 Issue 1- 2021

\author{
Author Details \\ Neil C Khoury*, Mehrdad Karimi and Gennadiy Grutman \\ Department of Internal Medicine, New York-Presbyterian Brooklyn Methodist Hospital, USA
}

*Corresponding author

Neil C Khoury, Department of Internal Medicine, New York-Presbyterian Brooklyn Methodist Hospital, Brooklyn, New York, 11215 , USA

Article History

Received: April 22, 2021 Accepted: April 30, 2021 Published: May 03, 2021

\begin{abstract}
Tuberculosis spondylitis, or "Pott's Disease", is a rare extrapulmonary manifestation of tuberculosis involving the spine. Very few cases of tuberculosis with sacral involvement have been reported, particularly in the United States, which may pose a diagnostic challenge for clinicians. We present a case report of extrapulmonary tuberculosis with sacral involvement in a 27 -year-old male who initially presented with systemic symptoms thought to be related to a hematologic malignancy.

Background: A total of 1.4 million people died from Tuberculosis (TB) worldwide in 2019. This infection can have devasting effects to the lungs and other organs if not treated. Cases of extrapulmonary TB may prove to be a diagnostic challenge. Tuberculosis spondylitis, or "Pott's Disease," is a rare extrapulmonary manifestation of TB involving the spine, seen in 1-3\% of TB cases [1]. Pott's disease most commonly affects the vertebral bodies of the lumbar or thoracic spine, and if left untreated, can lead to cord compression or spinal deformity [2]. Very few cases of TB with sacral involvement have been reported, particularly in the United States [3]. In fact, several large series reported no cases of tuberculosis with sacral involvement [3].
\end{abstract}

Keywords: Tuberculosis; Pott's disease; Sacral pott's; Tuberculosis spondylitis

\begin{abstract}
Abbreviations: TB: Tuberculosis; CT: Computed Tomography; ADA: Adenosine Deaminase; AFB: Acid Fast Bacilli; PCR: Polymerase Chain Reaction; MRI: Magnetic Resonance Imaging; RIF: Rifampin; INH: Isoniazid; PZA: Pyrazinamide; EMB: Ethambutol
\end{abstract}

\section{Objective}

We present a case report of extrapulmonary TB with sacral involvement in a 27 -year-old male who initially presented with systemic symptoms thought to be related to a hematologic malignancy.

\section{Case Report}

27-year-old male college student from Senegal with no significant past medical history presented to the emergency department for severe lower back pain for a few weeks. The pain was dull, 8/10 in intensity, radiating to the hips bilaterally, intermittent, progressively worsening, aggravated by ambulation, alleviated by lying flat and Acetaminophen. He also had unintentional weight loss of 40 pounds and night sweats for 2-3 months. He had no family history of malignancy. Vital signs on admission were within normal limits, except for tachycardia (heart rate
123 beats per minute). He was initially afebrile on admission, however, spiked a fever on day two of admission $\left(39^{\circ} \mathrm{C}\right)$. Physical examination was significant for decreased right lower lung breath sounds and moderate tenderness to palpation of the lower spine. Admission labs were significant for leukocytosis (white blood cell count 13,120uL), thrombocytosis $(568,000 \mathrm{uL})$, and elevated alkaline phosphatase 538IU/L. A Chest X-Ray showed a right pleural effusion with right compressive atelectasis. Computed Tomography (CT) scan of the chest with contrast showed a large loculated right pleural effusion and a pathological right pericardial lymph node. Thoracentesis with pleural fluid cytology analysis revealed an exudative fluid that was adenosine deaminase (ADA) positive. The pleural fluid cytology was negative for malignancy. CT scan of the abdomen and pelvis with contrast showed a destructive process centered on $\mathrm{S} 1$, with extensive lysis of the right hemi-sacrum (Figure 1). Further lab work including blood cultures, urine culture, tumor markers such as CEA, CA19-9, AFP, and testing for hepatitis and HIV, were all unremarkable. QuantiFERON testing was positive twice, however, six acid fast bacilli (AFB) sputum smears and one sputum mycobacterium TB complex DNA Polymerase Chain Reaction (PCR) were all negative. Magnetic resonance imaging (MRI) 
of the pelvis with and without contrast showed a large exophytic mass with aggressive bony destruction of S1 (Figure 2). Sacral biopsy revealed caseating granulomatous inflammation associated with giant cells and extensive necrosis and was negative for malignancy and AFB staining.

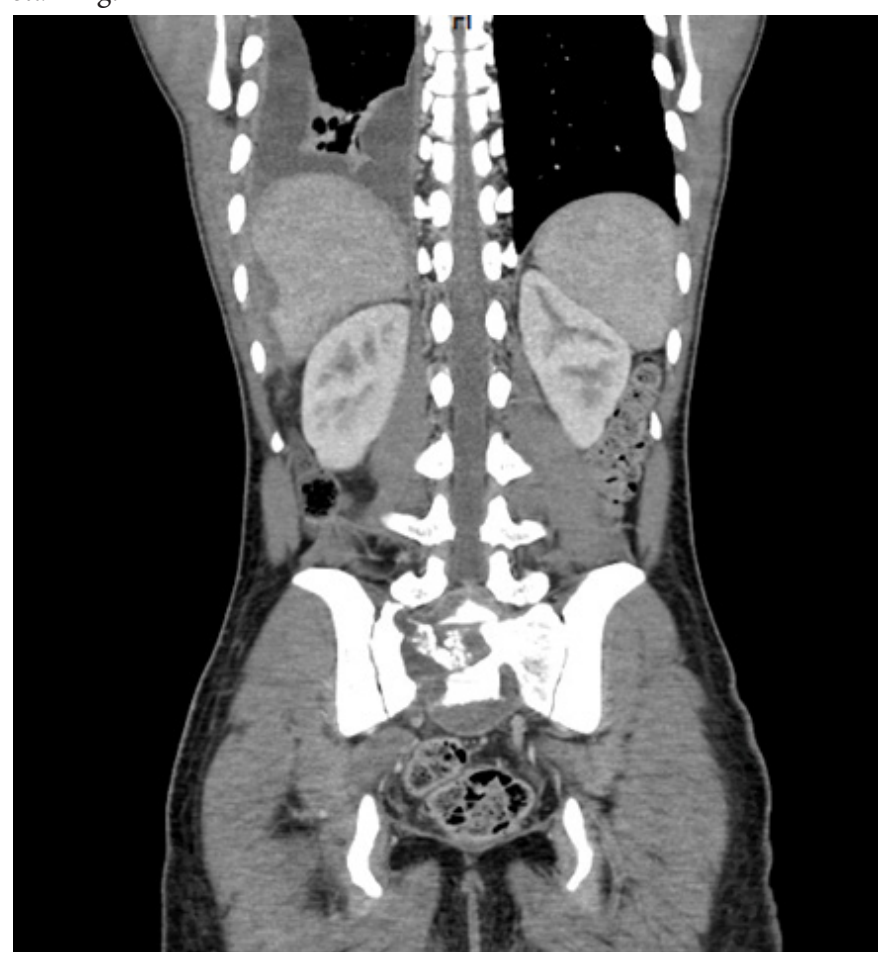

Figure 1: CT scan showing a destructive process centered on S1, with extensive lysis of the right hemi-sacrum.

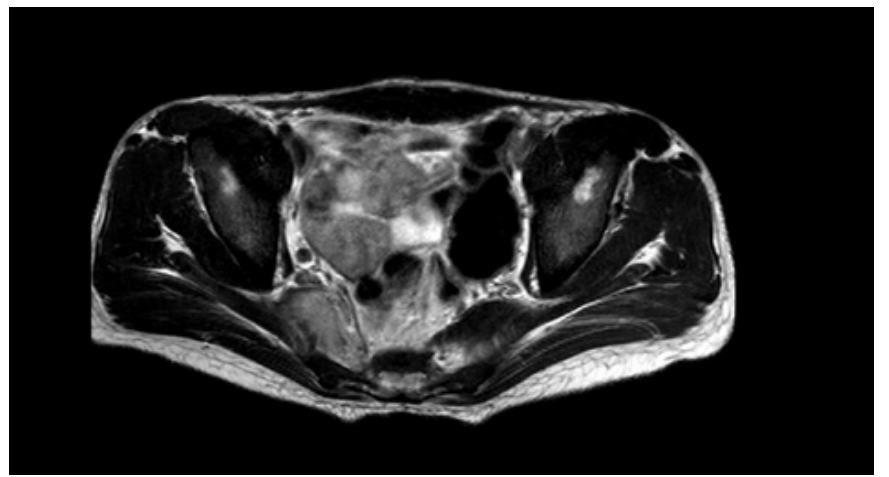

Figure 2: MRI (T1-weighted) showing a large exophytic mass with aggressive bony destruction of $\mathrm{S} 1$.

\section{Discussion}

Very few cases of TB with sacral involvement have been reported, particularly in the United States, which can pose a diagnostic challenge. Pott's disease would typically present with low-grade fevers, paraplegia, varying degrees of weakness, and potentially sensory involvement or spinal deformity $[4,5]$. National guidelines recommend obtaining three sputum samples for AFB smear analysis in diagnosing or ruling out pulmonary TB [6]. Studies show that sensitivity of a positive AFB smear increases from $67 \%$ to $71 \%$ and $72 \%$ with each consecutive AFB smear tested [6]. Sensitivity and specificity of elevated pleural fluid ADA for diagnosing a tuberculous pleural effusion has been reported up to $86-93 \%$ and $93-94 \%$ respectively [7]. For our patient, despite six AFB negative sputum samples, a negative sputum PCR, and an AFB negative sacral biopsy, ADA+ pleural fluid and a sacral biopsy finding of caseating granulomas were highly suggestive of tuberculosis. A biopsy sample was sent for mycobacterium tuberculosis culture. The patient was started on "RIPE" therapy; rifampin (RIF), isoniazid (INH), pyrazinamide (PZA), and ethambutol (EMB), and was discharged after a 15 days hospital course with instructions to follow up at the department of health pulmonary clinic or infectious diseases clinic at our institution in 1-2 weeks.

\section{References}

1. Varatharajah S, Charles YP, Buy X, Walter A, Steib JP, et al. (2014) Update on the surgical management of Pott's disease. Orthop Traumatol Surg Res 100(2): 233-239.

2. Turgut M (2001) Spinal tuberculosis (Pott's disease): its clinical presentation, surgical management, and outcome. A survey study on 694 patients. Neurosurg Rev 24(1): 8-13.

3. Khan SI, Ahmed N, Rahman A, Al Mahmud A, Islam R, et al. (2019) Tuberculous spondylitis involving sacrum-a rare location and presentation of Pott's disease: Case report and review of the literature. Bangladesh Journal of Medical Science 18(2): 440-445.

4. Pai M, Behr MA, Dowdy D, Dheda K, Divangahi M, et al. (2016) Tuberculosis. Nature reviews 2: 16076.

5. Dass B, Puet TA, Watanakunakorn C (2002) Tuberculosis of the spine (Pott's disease) presenting as 'compression fractures'. Spinal cord 40(11): 604-608.

6. Leonard MK, Osterholt D, Kourbatova EV, Del Rio C, Wang W, et al. (2005) How many sputum specimens are necessary to diagnose pulmonary tuberculosis? Am J Infect Control 33(1): 58-61.

7. Aggarwal AN, Agarwal R, Sehgal IS, Dhooria S (2019) Adenosine deaminase for diagnosis of tuberculous pleural effusion: A systematic review and meta-analysis. PloS one 14(3): e0213728. 